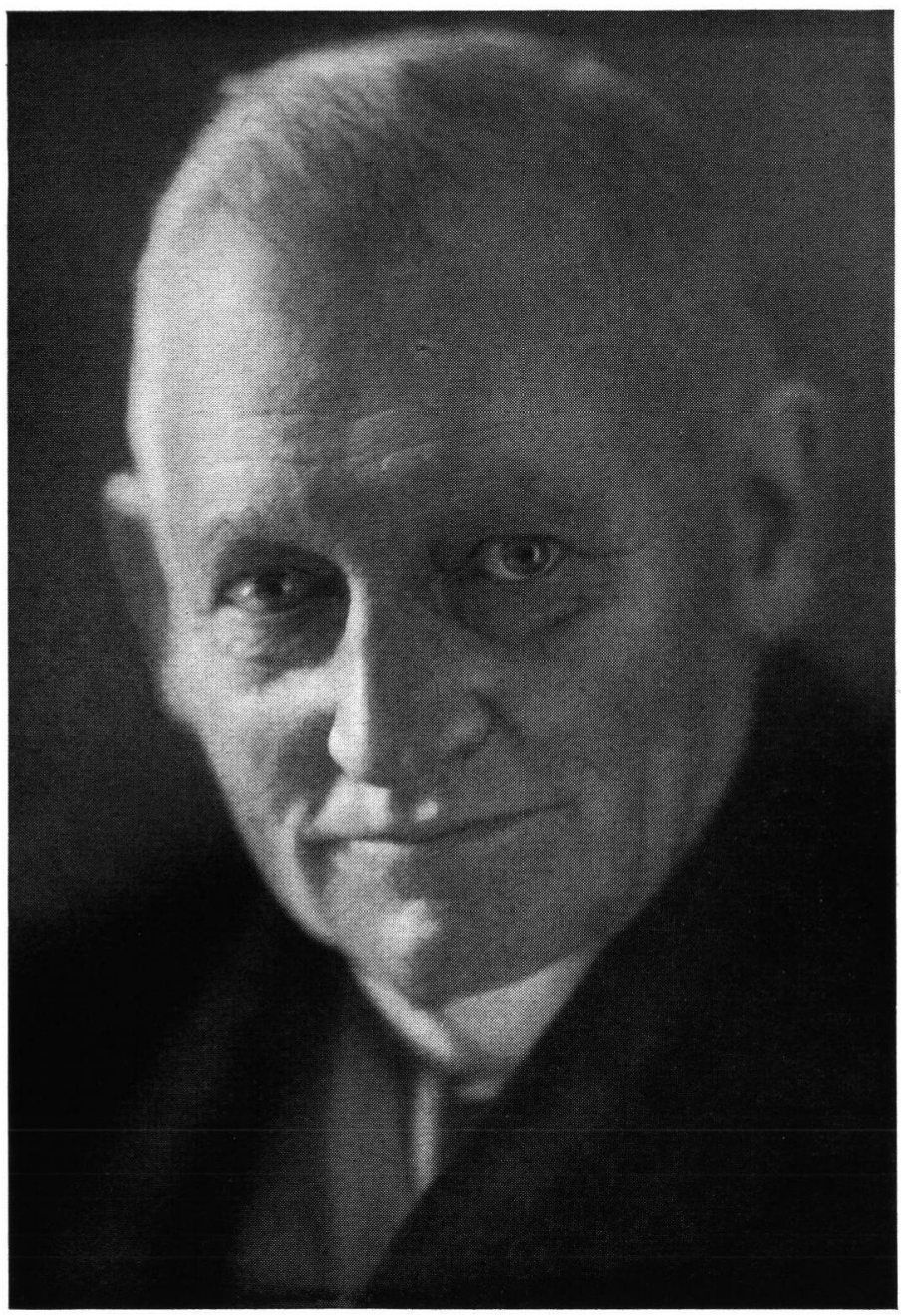

\title{
Albrecht Penck
}

geb. 25. September 1858 in Leipzig-Reudnitz

gest. 7. März 1945 in Prag-Reuth (Krč) 



\title{
Albrecht Penck zum Gedächtnis
}

\author{
Von Herbert Louis, München
}

Hierzu das Titelbild (Taf. I)

Am 25. September jährt sich zum hundertsten Male der Geburtstag von Albrecht Penck. Das ist für alle Quartärforscher und für alle Geographen ein Anlaß, die Gedanken zu diesem großen Gelehrten zurückzulenken und ihn und sein Schaffen aus dem Abstand des Heute zu überdenken. Wer dies versucht, wird sich einer erstaunlichen Vielseitigkeit und Nachhaltigkeit seines Wirkens bewußt. Zur richtigen Würdigung PENcks erscheint es notwendig, gerade die Weite seines Tätigkeitsfeldes hervorzuheben. Er ist gewiß ein überragender Eiszeitforscher gewesen. Aber sein Schaffen greift über den Interessenkreis der Quartärforschung weit hinaus.

Der junge Mann, der mit 19 Jahren seine erste Publikation über nordische Basalte im Diluvium von Leipzig erscheinen ließ, vollbrachte 1879 mit 21 Jahren durch seine Arbeit über „Die Geschiebeformation Norddeutschlands“ seine erste wissenschaftliche Großtat. In einer Zeit, als die Driftheorie von LyelL (1835) und die Vergletscherungstheorie von SEfström (1836) und Torell (1875) zur Erklärung der Geschiebelehme Norddeutschlands noch ernst miteinander rangen, entkräftete er die Driftheorie endgültig, sicherte er auf Grund eigener Anschauung und durch neue vertiefte Deutung älterer Beobachtungen die Vergletscherungstheorie zuverlässig, und brachte er schwerwiegende Beweisgründe bei für eine nicht nur einmalige, sondern mindestens zweimalige, wahrscheinlich dreimalige Vergletscherung Norddeutschlands. In der Rückschau muß betont werden, daß nicht nur die Tatsache der Mehrzahl der Eiszeiten selbst, sondern namentlich auch die wesentlichsten Feststellungen, auf Grund deren PENCK damals die Mehrheit der Eiszeiten begründete, aller späteren Kritik standgehalten haben. So gebührt ihm das Verdienst, den ersten umfassenden Beweis für den Polyglazialismus in Norddeutschland erbracht zu haben.

Drei Jahre später, im Jahre 1882, folgte seine Münchener Habilitationsschrift über „Die Vergletscherung der Deutschen Alpen, ihre Ursachen, periodische Wiederkehr und ihr Einfluß auf die Bodengestaltung ", die zugleich von der Sektion Breslau des Deutschen und Österreichischen Alpenvereins als Preisschrift gekrönt wurde. In ihr ist die Erkenntnis des mehrfachen Wechsels von Eiszeiten und Interglazialzeiten für das deutsche Alpengebiet mit einer bewundernswerten Fülle eigener und fremder Beobachtungen begründet worden. Daneben sind aber weitreichende Einsichten über Wesen und Formenschatz der glazialen Erosion und Akkumulation gewonnen, die der damals erst tastenden Glazialmorphologie fundamentale Erscheinungszusammenhänge offenbarten. Dieser großartige Anfang wurde von 1901 bis 1909 ausgebaut und auf den Gesamtbereich der Alpen ausgedehnt zu dem gemeinsam mit Ed. BrüCKNER geschaffenen Riesenwerk über „Die Alpen im Eiszeitalter".

Heute, nach einem halben Jahrhundert, bildet dieses immer noch die Grundlage der weiteren Forschung in seinem Bereich. Denn obschon einzelne Annahmen dieses Werks sich später als nicht haltbar erwiesen haben, wie z. B. die einer übergroßen Glazialerosion, sind doch im ganzen die unglaublich reichen Beobachtungen derart verläßlich und bezüglich ihrer Aussagekraft so sicher abgewogen, daß das kühne und weitreichende Ideenund Lehrgebäude, das PENCK auf Grund seiner Erfahrungen errichtete, sich in den wesentlichsten Zügen als durchaus standfest erwiesen hat. Viel zu wenig wird von den Nachfolgenden meist gewürdigt, mit wie unvollkommenen Karten PENCK seine Feldbeobach- 
tungen durchführen mußte. Auch ist es gut, sich gegenwärtig zu halten, daß ihm damals außer seiner Marschfähigkeit an Beförderungsmitteln nur die Eisenbahn und ausnahmsweise einmal ein Pferdewagen zur Verfügung stand. Das Werk hat die Anregung zu zahlreichen weiteren Forschungen gegeben und tut dies auch heute noch. Seine Hauptergebnisse sind dadurch verfeinert und bereichert worden. Aber sie wurden nicht umgestoßen.

Die Eiszeitforschung hat PENCK sein Leben lang nicht wieder losgelassen. Neben kleineren Reiseergebnissen aus Norwegen (1892), Großbritannien (1883-97), den Pyrenäen (1897), aus Canada (1898), der Balkanhalbinsel (1900), Australien (1900) aus älterer Zeit, entstanden in den zwanziger und dreißiger Jahren höchst wichtige Spezialforschungen über die Umgebung von Mittenwald (1922-28), die Höttinger Breccie und das Inntal (1921), außerdem glänzende Studien über die Einordnung des prähistorischen Menschen in den Ablauf des Eiszeitalters (1938) sowie eine Art Länderkunde des eiszeitlichen Europa, die der weiteren Forschung auf diesem Gebiet den Weg gewiesen hat.

Neben der Eiszeitforschung, vielfältig mit ihr verknüpft, aber doch weit über sie hinausstrebend, beginnt bei PENCK frühzeitig, mindestens seit 1882, die Auseinandersetzung mit der Geomorphologie im ganzen. Schwankungen des Meeresspiegels (1882), Theorien über das Gleichgewicht der Erdkruste (1889), Gesetzmäßigkeiten der Gebirgsverbreitung (1890), Einfluß des Klimas auf die Gestalt der Erdoberfläche (1883), Periodizität der Talbildung (1884), Denudation der Erdoberfläche (1887), die Bildung der Durchbruchstäler $(1888 ; 1890)$, das Endziel der Erosion und Denudation (1889), das sind Gegenstände, die ihn beschäftigten, bevor er 1894 seine zweibändige „Morphologie der Erdoberfläche" herausbrachte.

Dieses Werk ist ebenso hervorragend durch seine Literaturbeherrschung wie durch die Gestaltungskraft, mit der ein höchst komplizierter Stoff übersichtlich und folgerichtig dargeboten wurde, wie endlich durch die Strenge der Begriffsbildung und der wissenschaftlichen Aussage. Es stellte dem essayartigen „Führer für Forschungsreisende“ von Ferdinand von Richthofen (1886) eine umfassend-systematische Behandlung der Geomorphologic an die Seite. Das Werk ist auch heute, mehr als 60 Jahre nach seinem Erscheinen, trotz der stürmischen Weiterentwicklung der Geomorphologie, vor allem in der Erörterung der Grundprinzipien, nicht entbehrlich geworden. Manche seiner Anregungen, so die einer strenger physikalischen Erfassung geomorphologischer Vorgänge, sind noch längst nicht ausreichend verwirklicht.

Kleinere, aber sehr ergebnisreiche Feldforschungen und theoretische Arbeiten zur Geomorphologie haben PENCK neben seinen Glazialstudien auch in den späteren Jahren dauernd beschäftigt, so vor allem: Die Talgeschichte der obersten Donau (1899); Das Durchbruchstal der Wachau und die Lößlandschaft von Krems (1903); Geomorphologische Studien aus der Herzegowina (1900); Das Karstphänomen (1904); Das unterirdische Karstphänomen (1924); Der Drakenberg und der Quathlambabruch in Südafrika (1908); Die Morphologie der Wüsten (1909); Klimaklassifikation auf physiogeographischer Grundlage (1910); Die Formen der Landoberfläche und Verschiebungen der Klimagürtel (1913); Gekippte Seen (1924); Die Gipfelflur der Alpen (1919); Das Antlitz der Alpen (1924); Geomorphologische Probleme im fernen Westen Nordamerikas (1929).

Die außerordentlichen Forschungserfolge, die schon der junge PENCK auf den Gebieten der Eiszeitkunde und Geomorphologie errungen hatte, führten bereits 1885 zur Berufung des 27-Jährigen auf die ordentliche Professur in Wien. Damit ergab sich für ihn ein sehr großer neuer Aufgabenkreis. Er kommt in seinem literarischen Schaffen u. a. zum Ausdruck durch die Inangriffnahme länderkundlicher Werke. 1887 erscheint „Das Deutsche Reich“, 1889 die Darstellung der Niederlande, Belgiens und Luxemburgs in der KIRCHHOFFschen Länderkunde, kleinere Schriften über österreich-ungarische Gebiete folgen. Sind diese Arbeiten, die neben den Feldforschungen einherliefen, auch nicht die eigentliche 
Ursache seines großen wissenschaftlichen Ruhmes, so waren sie doch für die Jahrzehnte nach ihrem Erscheinen Standardwerke. Auch in heutiger Sicht sind sie weit mehr als nur Zeugen von PENCKS unerhörter Arbeitskraft. Es sind methodisch wichtige Marksteine auf dem Wege zur wissenschaftlich-geographischen Länderkunde. Es lag an der damaligen Gesamtentwicklung der Geographie, wenn in ihnen im wesentlichen nur die physischen Aspekte der Länder einer streng genetisch deutenden Darstellung teilhaftig wurden, ja wenn hierbei sogar ïber die eigentlich länderkundliche Zielsetzung hinausgegangen wurde, während die Zusammenhänge auf anthropogeographischem Gebiet nicht in gleicher Weise durchleuchtet werden konnten. Aber das in ihnen allenthalben deutliche Streben nach Erfassung der Zusammenhänge hat als Vorbild mächtig gewirkt.

Erst in späteren Jahren hat sich PENCK dann wieder länderkundlichen Gegenständen zugewandt, freilich nur in kürzeren Aufsätzen. Aber unter ihnen sind Arbeiten von bleibendem Wert, dic die tiefe Erkenntnis ausgereiften geographischen Sehens offenbaren, wie „Der Großgau im Herzen Deutschlands“ (1921); „Deutschland als geographische Gestalt" (1926); „Finnland“ (1927) und „Finnlands Natur“ (1927); „Zwischeneuropa“ (1916); „Central Asia“ (1930).

Sie stellten sich ein, als sich der an der Schwelle des Alters stehende Mann nach den Erlebnissen des ersten Weltkrieges auch anthropogeographischen Problemen ernst zuwandte. Die Frage der (Bevölkerungs-)Tragfähigkeit der Länder (1926), das Hauptproblem der Physischen Anthropogeographie (1924), die Bonitierung der Erdoberfläche (1926) einerseits, der Gedanke des Deutschen Volks- und Kulturbodens (1925) andererseits werden von ihm aufgerollt. Es ist eine neue, noch ungewohnte Art, mit der der naturwissenschaftliche Beobachter PENCK diese Gegenstände sieht und angreift. Sie hat eine ganze Literatur ins Leben gerufen, die zum mindesten hinsichtlich des ersten Fragenkreises dauernd weiter anwächst.

Frühzeitig hat sich PENCK auch Fragen der Kartographie zugewandt. Seit 1891 hat er für die Herstellung einer einheitlichen Weltkarte 1:1 Mill. gekämpft. Das tatsächliche Ingangkommen dieses großen internationalen Unternehmens war zu erheblichem Teil sein Erfolg und war der geographischen Wissenschaft ungemein förderlich. Nicht zu Unrecht ist von geodätischer Seite vermerkt worden, daß vermeidbare Mängel in den mathematischen Grundfestsetzungen dieses Kartenwerks vorhanden sind. Wer aber um die Verhandlungsschwierigkeiten in internationalen Gremien weiß, der wird trotz dieser Schönheitsfehler den außerordentlichen Gewinn dankbar anerkennen, den die Verwirklichung des Plans gegen Skepsis und Widerstand vieler Fachleute erfahrenen Benutzern von Karten auch kleiner Maßstäbe ohne Zweifel brachte. Ein solcher war PENcK. Als solcher, nicht als geodätischer oder kartographischer Fachmann, hat er auch zur Vollendung der Karte des Deutschen Reiches 1:100000 Stellung genommen und die Pflege hochwertiger topographischer Spezialkarten (Schrammsteinkarte), ganz besonders der Hochgebirgskarten und Hochgebirgsreliefs des Alpenvereins, gefördert. Sein ideeller Anteil an der Schaffung dieser wissenschaftlich wie allgemein kulturell gleich hochwertigen kartographischen Leistungen ist sicherlich sehr bedeutend gewesen.

Nur selten hat PENCK zu Fragen der Methodik und der theoretischen Fundierung seiner Wissenschaft das Wort ergriffen. Ihm schien es wichtiger, mit Hilfe klarer Begriffsbildungen methodisch gute Forschungsarbeiten $\mathrm{zu}$ verfassen, als über Methodik zu schreiben. „Die Physiographie als Physiogeographie in ihren Beziehungen zu anderen Wissenschaften“ (1905), „Beobachtung als Grundlage der Geographie“ (1906), „Die Geographie unter den erdkundlichen Wissenschaften“ (1928) und „Neuere Geographie“ (1928) sind wohl seine wichtigsten Außerungen auf diesem Gebiet.

In ihnen betont er, von der Auffassung Ferdinands von Richthofen von der Geographie als Wissenschaft von der Erdoberfläche ausgehend, die Einheit der geographicchen 
Wissenschaft im ganzen und handelt von der Wesensbestimmung und Abgrenzung der Geographie gegen die Nachbarwissenschaften. Er erläutert an Beispielen die Erfolge der systematischen Beobachtung in der geographischen Forschungsarbeit.

Der bedeutendste und lebendig weiterwirkende Gedanke dieser Arbeiten scheint mir in Pencks Hinweis auf die einzigartige Wichtigkeit der Erdoberfläche als den eigentlichen Ort der großen Umsetzung der zugestrahlten Sonnenenergie zu liegen, welch letztere die wesentliche Ursache oder wenigstens die wesentliche Voraussetzung so gut wie allen irdischen Geschehens ist. Dieser Gedanke verleiht erst von Richthofens Lehre von der Geographie als Wissenschaft von der Erdoberfläche eine für physische Geographie, Biogeographie und Anthropogeographie gleich verbindliche und gleich großartige einheitliche Sinngebung. Er klingt schon in PeNCKs Schrift von 1905 an und ist 1928 mit aller Klarheit herausgearbeitet. Er hat das theoretische Fundament der Geographie als Gesamtwissenschaft ungemein gefestigt.

Unter PENCKs Schriften finden sich einige Polemiken. Er hat in solcher Weise nur dann das Wort genommen, wenn wohlbegründete, wichtige Ergebnisse seiner Arbeit oder seines Tuns, wie mehrfach geschehen ist, auf Grund haltloser Argumente, aber mit dem Schein des autoritativen Sachverständnisses angegriffen worden waren. In solchen Fällen hat PencK, wie etwa in dem Aufsatz „Richard Lepsius über die Einheit und die Ursachen der diluvialen Eiszeit in den Alpen" (Zeitschr. f. Gletscherkunde 1912, S. 161-189), die Schwächen der gegnerischen Außerungen mit trockenem Sarkasmus gründlich und schonungslos bloßgelegt. Seine wissenschaftlichen Widersacher haben ihm dies zweifellos verübelt. Aber man wird PENCKs Vorgehen sachlich wie menschlich verstehen müssen. Er hat begründete Kritik seiner Anschauungen immer gelten lassen und gewürdigt. Aber es war notwendig für ihn, gediegene Früchte seiner mit Aufopferung vollbrachten, ernsten Arbeit gegen Gefährdung durch Unkenntnis, wenn diese sich fachmännisch gebärdete, wirkungs-voll zu schützen. Dem Nachfahren vermitteln diese Beispiele einer ebenso eleganten wie vernichtenden Auseinandersetzung mit anmaßend-unbedachten Gegnern einen Hauch der wirklichen Überlegenheit, die PENCK besaß.

Noch über den in Pencks Werken enthaltenen Forschungs- und Denk e $\mathrm{r} g$ e b $\mathrm{n}$ is s e $\mathrm{n}$ steht die formende Nachwirkung seiner Forschungs- und Arbeits we is e. Sie mag besonders lebhaft bei denen sein, die ihn gekannt haben und seiner Persönlichkeit tiefer bewußt geworden sind. Aber sie teilt sich auch denjenigen mit, die seine Werke nachträglich studieren. Aus einer Zeit stammend, in der noch kompendienhaftes Zusammenstellen von Tatsachen auf geographischem Gebiet für Wissenschaft angesehen wurde, hat PENCK in seinen Feldforschungen von Anfang an, später auch auf den anderen Gebieten der Geographie, namentlich auch in der Länderkunde, nur solches Bemühen als wissenschaftlich gewertet und selbst betätigt, das der Verfolgung echter Probleme diente.

Probleme zu sehen und ihnen mit Hilfe von Beobachtung nachzugehen, das war sein Tun auf dem Gebiet der Wissenschaft während seines ganzen Lebens. Worin aber dieses Beobachten besteht, das hat er nicht einmal in seiner Berliner Antrittsvorlesung mit dem Titel „Beobachtung als Grundlage der Geographie“ (1906) genauer umrissen. Es entsprach offenbar so sehr seiner Natur, daß er es nicht für erläuterungsbedürftig erachtete. Wenn man versucht, das Wesen dieses Beobachtens zu umreißen, so läßt sich vielleicht folgendes sagen: PENCK besaß in wohl ganz außergewöhnlichem Maße die Gabe, sich von der umgebenden Welt auf Grund seiner Wahrnehmungen dauernd ein weithin durchgezeichnetes, rational überlegtes, geistiges Bild zu machen. Indem er dieses mit neu hinzukommenden Wahrnehmungen ständig verglich, wurde er auf Unstimmigkeiten zwischen gewissen seiner Wahrnehmungen und seinem geistigen Bilde aufmerksam. Das waren die Probleme. Um sie zu lösen, mußte unter Berücksichtigung der Naturgesetze und der Logik ein solcher Umbau der geistigen Vorstellungen vorgenommen werden, daß hernach a $11 \mathrm{e}$ Wahrnehmungen gemeinsam widerspruchslos darin Platz fanden, möglichst auch solche, 
die später noch neu auftauchten oder sogar erst mit Hilfe der verbesserten Allgemeinvorstellung aufzufinden waren.

PENCK sah einen wichtigen Schlüssel zu sehr vielen verzwickt erscheinenden Einzelfragen in der sorgfältigen Beachtung örtlicher Sondergegebenheiten, also in einer echt geographischen Blickrichtung. Er zeigte, wie oft die Sicherheit allgemeiner Erkenntnisse dadurch gesteigert wird, daß scheinbare Unstimmigkeiten als zwangsläufige Folgen örtlicher Sonderverhältnisse erklärbar werden, und wie fast kein geographisches Problem ohne die Berücksichtigung derartiger Besonderheiten wirklich aufgehellt werden kann.

Das zu solcher Arbeitsweise nötige lebhafte Betätigen der wissenschaftlichen Phantasie unter nüchterner Kontrolle durch naturwissenschaftliche Geisteshaltung und durch den sorgfältigen Vergleich mit den sachlichen Gegebenheiten, das war Beobachten in seinem Sinne, nicht etwa mechanisches Registrieren und Klassifizieren von Erscheinungen nach vorgefaßtem Gedankenschema.

Aus solcher Geisteshaltung erwuchsen seine erstaunlichen Forschungserfolge, ebenso auch seine oft kühnen Hypothesen und Annahmen, die er aber fallen ließ, sobald entscheidende Gegengründe sichtbar wurden. Die Zahl der Irrtümer, die er auf wissenschaftlichem Felde begangen hat, ist, gemessen an den großen und echten Erkenntnissen, die er uns schenkte, recht klein, und es muß hinzugefügt werden, daß er nach gewonnener Einsicht von ihnen abrückte.

Am Fortgang seiner Forschungsarbeit ließ er seine Schüler, Verehrer und Freunde offen teilnehmen. Sie haben viel davon gewonnen, und gerade diese Art seines Schaffens hat seinen Einfluß als Lehrer des Forschens ebenso wie als Erzieher sehr groß gemacht. Die Schar derer, die sich ihm in solcher Weise verbunden und verpflichtet fühlen, dürfte auch gegenwärtig um die Zeit seines hundertsten Geburtstages noch sehr groß sein.

Ergänzende Schriften über A. PENCK und sein Werk:

1877-1928 Druckschriften von Albrecht PeNCK, zusammengestellt zu seinem 70. Geburtstag. - Als Manuskript, Berlin 1928.

A. Penck: Sechzig Jahre Eiszeitforschung (Eine Art Selbstbiographie). - In „Die Erde“, Heft 1, S. 5-11. Berlin 1949.

N. Krebs: Albrecht Penck, eine Skizze seines Wirkens (zum 70. Geburtstag). - Velhagen u. Klasings Monatshefte, S. 181-184, 1928.

W. Behrmann: Albrecht Penck. Zum 70. Geburtstag des hervorragenden Geographen. - Westermanns Monatshefte 1928, S. 201-204.

W. Behrmann: Die Bedeutung Albrecht Pencks für die Kartographie. - Bibliographisches Institut, Leipzig 1938.

N. Krebs: Nachruf auf Albrecht Penck. - Jahrb. d. Deutsch. Akad. d. Wiss. Berlin 1946-1949, S. 202-212. 1949.

H. Louis: Albrecht Penck, der bahnbrechende Eiszeitforscher. - In „Forscher und Wissenschaftler im heutigen Europa“, S. 279-288. Oldenburg 1956.

Manuskr. eingeg. 9. 11. 1957.

Anschrift des Verf.: Prof. Dr. Herbert Louis, München 2, Luisenstraße 37, Geograph. Institut der Universität. 